\section{Hipersensibilidade a fungos em crianças asmáticas de uma comunidade do Recife, Pernambuco}

\section{Hypersensitivity to molds in asthmatic children from a community of Recife, Pernambuco}

Ana Carla de Albuquerque Osório 1

Nilza Rejane Sellaro Lyra 2

Emanuel Sávio Cavalcanti Sarinho 3

1-3 Centro de Pesquisas em Alergia e Imunologia em Pediatria. Hospital das Clínicas. Universidade Federal de Pernambuco. Av. Prof. Moraes Rego, s. n. Cidade Universitária. Recife, PE, Brasil. CEP: 50.670-420 E-mail: nilza.lyra@gmail.com

\section{Abstract}

Objectives: to identify prick test hypersensitivity to molds in asthmatic school children in a low-income urban community.

Methods: thirteen children who had more than three asthma attacks in the last 12 months from March'1997 to June'1998 were evaluated. These children were selected from a previous cross-sectional survey studying 123 children from 6 to 10 years old residing at the low-income urban community of Campo do Banco-Várzea, Recife, Pernambuco. The 123 children were tested by the International Study of Asthma and Allergies in Childhood survey to determine asthma. The 13 asthmatic children selected were evaluated with the prick test for immediate hypersensitivity to six standard mold extracts: aspergillus mix, penicillium mix, hormodendrum cladosporidiodes, alternaria tenius, helminthosporium interseminatum and mold mix.

Results: there were 12 mold-sensitive children from the group of 13 children, showing a positive result to at least one of the mold extracts used (12/13). Mold extracts of higher positive incidence were: Aspergillus mix (7/13), Penicillium mix (6/13) and Hormodendrum Cladosporidiodes (5/13).

Conclusions: the evaluated children had high prick test reactivity to mold extracts. The findings indicate the need for additional studies to analyze a possible association between fungal exposure and asthma.

Key words Asthma, Fungi, Skin tests

\section{Resumo}

Objetivos: identificar a sensibilização a testes cutâneos de hipersensibilidade imediata para fungos em crianças asmáticas, residentes em comunidade urbana de baixa renda.

Métodos: no período de março de 1997 a junho de 1998 foram avaliadas 13 crianças com mais de três episódios de dispnéia nos últimos 12 meses, selecionadas a partir de um estudo transversal, em que todos os 123 escolares de 6 a 10 anos residentes na comunidade responderam ao questionário International Study of Asthma and Allergies in Childhood. Nas 13 crianças com asma em atividade, foram aplicados testes cutâneos de hipersensibilidade imediata para avaliar resposta a seis extratos fúngicos padronizados: Aspergillus mix, Penicillium mix, Hormodendrum cladosporidiodes, Alternaria tenius, Helminthosporium interseninatum e Mold mix.

Resultados: dentre as 13 crianças analisadas, 12 apresentaram sensibilididade a pelo menos um dos fungos testados (12/13), cujos extratos com maior frequência de positividade foram: Aspergillus mix (7/13), Penicillium mix (6/13) e Hormodendrum cladosporidiodes (5/13).

Conclusões: a freqüência elevada de hipersensibilidade aos extratos de fungos nas crianças avaliadas sugere a necessidade de estudos analíticos observacionais para esclarecer uma possível associação causal entre fungos e asma.

Palavras-chave Fungos, Asma, Testes cutâneos 


\section{Introdução}

Estudos epidemiológicos sobre as doenças alérgicas, em particular a asma, têm auxiliado a compreender melhor a complexa interação entre a predisposição genética e os fatores ambientais na gênese dessas doenças. ${ }^{1}$ Dentre os alérgenos presentes na poeira domiciliar, merecem destaque: os ácaros, epitélios de animais, os antígenos de barata e os fungos. ${ }^{1}$ Há evidências de que exposição ambiental ao mofo ou derivados protéicos desses, pode desencadear alergia IgE -mediada, notadamente asma, em indivíduos predispostos. 2 Também há relatos de que tais alérgenos podem ser importantes em casos de dermatite atópica, funcionando como o "gatilho" desencadeante para a doença inflamatória da pele em tais pacientes. 3

Em estudo realizado na França, foi observado que proteínas oriundas de diversos gêneros de fungos presentes em domicílios de asmáticos eram responsáveis por 14,6\% de sensibilização entre tais indivíduos, precedidos pelos ácaros da poeira domiciliar e os epitélios de animais, evidenciando assim a importância dos fungos na patogênese da asma. 4 Pela complexidade alergênica, é consenso geral que existem muitas dificuldades no diagnóstico de hipersensibilidade a fungos, pois há necessidade de controle extremamente rigoroso na procedência e qualidade do extrato. 4 Contudo, a hipersensibilidade ao mofo, apesar da não disponibilidade de teste cutâneo ou de $\operatorname{IgE}$ específica perfeitamente padronizada na prática clínica, tem sido incriminada nos casos rebeldes de asma e incluída entre as estratégias de prevenção.5,6 Em pesquisas científicas, quando os testes cutâneos com extratos adequados são positivos, o tratamento hipossensibilizante para um determinado gênero de fungo tem sido considerado e tem apresentado resultados satisfatórios. 5-8

Quando comparadas às pesquisas sobre exposição com estudos de sensibilização a mofo domiciliar, há uma diferença nos resultados, indicando a complexidade do assunto. Em estudo de exposição, Guneser et al. ${ }^{9}$ na Turquia, em 1994, realizaram a coleta gravitacional em placas de Petri no ambiente, em que 26 espécies de fungos foram isoladas, sendo o gênero Penicillium o mais freqüente $(29,6 \%)$, seguido por Mucor (23,9\%), Aspergillus niger (22,2\%), Rhizopos (19,8\%) e Alternaria (19,8\%). Em ambiente domiciliar na Polônia, Penicillium e Aspergillus foram os gêneros mais comumente encontrados.10 Em relação aos estudos de sensibilização, o gênero Alternaria foi identificado como o principal fungo causador de hipersensibilização na
Espanha, 11 enquanto na Dinamarca12 o Cladosporium tem ocupado lugar de destaque. Portanto, existem vários relatos de sensibilização, o que indica, de um modo geral, que a prevalência de sensibilização a fungos vem aumentando. No Japão, desde a década de 1960 já tinha sido observado um aumento de positividade nas crianças asmáticas aos testes cutâneos para fungos. 13

Em estudos feitos no Brasil, no estado de Minas Gerais, ${ }^{14}$ foram identificados no ambiente domiciliar os seguintes fungos, por ordem de freqüência: Cladosporium (90,3\%), Penicillium (64,7\%), Aspergillus (58,6\%), Curvularia (33,3\%), Pullularia $(31,9 \%)$ e Epicoccum (31\%). Foi também utilizado o teste cutâneo de hipersensibilidade, quando pela primeira vez encontrou-se freqüência elevada de sensibilização ao gênero Alternaria $(21,1 \%)$ fato que, até então, não havia sido relatado no Brasil.14 Outro estudo para avaliar hipersensibilidade ao mofo, realizado no estado de São Paulo, 15 demonstrou baixa frequiência de positividade aos testes cutâneos para fungos (extratos de Aspergillus, Cladosporium, Penicillium, Rhizopus e Mucor) em pacientes com asma e ou rinite, apesar de nos pacientes apenas com asma os índices serem discretamente mais elevados $(7,28 \%)$ do que nos pacientes com rinite isolada $(5,75 \%)$.

Em Recife, 16 em 1958, foram identificados por ordem de freqüência de isolamento no ambiente os gêneros: Aspergillus, Penicillium, Fusarium, Curvularia e Candida. Posteriormente, em 12 áreas nessa cidade, em 1979 e em 1983, verificou-se que os fungos mais freqüentemente isolados no ar atmosférico foram: Aspergillus (58,9\%), Penicillium (41,4\%), Cândida sp. (30,6\%), Cladosporium (20,8\%), Fusarium (19,6\%), Phoma (19,4\%) e Curvularia $(18,9 \%) .17$

O presente estudo foi realizado visando identificar a sensibilização aos testes cutâneos de hipersensibilidade imediata para fungos em crianças asmáticas na faixa etária de 6 a 10 anos, residentes na comunidade de Campo do Banco, no bairro da Várzea, Recife, Pernambuco.

\section{Métodos}

Baseado nos achados dos estudos anteriormente citados foi realizado um projeto piloto previamente a este estudo, em residências de crianças asmáticas do Grande Recife, no ano de 1997, tendo sido utilizado o método de coleta por gravidade através de placas de Petri. Na ocasião foi observada a maior freqüência dos seguintes espécimes de fungos nos 
referidos domicílios: Aspergillus (15,15\%); Cladosporium $(10,30 \%)$; e Penicillium $(8,48)^{18}$ evidenciando então a importância de avaliar a possibilidade de sensibilização.

O estudo foi desenvolvido a partir do projeto piloto, pela necessidade de relacionar a possível hipersensibilidade a tais fungos em pacientes com asma em atividade. Foram selecionados os três espécimes mais encontrados no ambiente para a realização de testes cutâneos de hipersensibilidade imediata utilizando extratos padronizados.

A coleta de dados e a realização dos testes alérgicos ocorreram no período de março de 1997 a junho de 1998. A população estudada foi composta por todas as crianças de 6 a 10 anos, residentes em Campo do Banco, bairro da Várzea, na cidade do Recife, Permambuco, Brasil, uma comunidade de baixa condição socioeconômica. Por ocasião da coleta dos dados, observou-se que na quase totalidade das residências predominavam esgoto a céu aberto e depósito de lixo próximo aos domicílios. A comunidade é assistida pelo referido Hospital das Clínicas da Universidade Federal de Pernambuco, o que confere maior confiabilidade aos dados pelo prévio acompanhamento médico. A proximidade do hospital facilitou o deslocamento das crianças e responsáveis para a realização dos testes alérgicos com antígenos sabidamente complexos como os fungos. A faixa etária escolhida (6 a 10 anos) justifica-se devido ao fato de que as crises de asma antes dos cinco anos de idade são, em geral, precipitadas por vírus, e porque apenas a partir dessa idade os alérgenos ganham importância na etiopatogenia. 19

Foi realizado estudo descritivo de corte transversal com as 123 crianças submetidas ao questionário do International Study of Asthma and Allergies in Childhood (ISAAC) para identificação de portadoras de sintomas de asma. ${ }^{20}$ Foram recrutadas todas aquelas que apresentaram mais de três crises de dispnéia, nos últimos 12 meses, obtendo-se assim um subgrupo de 13 crianças, as quais foram encaminhadas para unidade hospitalar de grande porte - Hospital das Clínicas da Universidade Federal de Pernambuco, para o Ambulatório Especializado de Asma e Alergia da Disciplina de Pediatria.

Conforme já mencionado, foi usado como instrumento de pesquisa o questionário utilizado no ISAAC, o qual foi aplicado pelo pesquisador em visita domiciliar, entrevistando a genitora ou o adulto responsável por cada criança. No formulário, também foram incluídas perguntas sobre condições socioeconômicas, historia pessoal e familiar de alergia/asma, exposição a fungos e outros alérgenos e ainda sobre possíveis agravantes de asma como exposição ao fumo e aos poluentes.

Os testes cutâneos foram realizados pela pesquisadora (ACAO) após obtenção do termo de consentimento livre e esclarecido do responsável, em ambiente hospitalar com capacidade de oxigenação e medicação de urgência. Foram utilizados extratos padronizados dos seguintes fungos: Alternaria tenius, Aspergillus mix, Penicillium mix, Hormodendrum cladosparidiodes, Helminthosporium interseminatum, Mold mix A (Rhizopus nigrans, Fuzarium vasinfectum, Phoma herbarum, Mucor racemosus, Aspergillus mix, Batrytis cinerea, Chaetomium indicum, Epicoccum nigrum, Geotrichum candidum, Helminthosporium interseminatum, Monilia sitophilia, Penicillium mix, Pullularia pullulans, Rhodotorula nigricans, Saccharomyces cerevisiae), especialmente elaborados para esta pesquisa, procedentes do laboratório Bayer Corporation (USA).

Os testes cutâneos (prick testes) de hipersensibilidade imediata foram realizados por puntura na superfície interna do antebraço, onde eram colocadas uma gota de cada antígeno específico dos vários fungos, solução salina (controle negativo) e histamina (controle positivo). Foram usados puntores descartáveis em uma rotação epicutânea de 360 graus, com a distância entre os testes de, no mínimo, dois centímetros. Após a puntura, esperava-se 15 minutos para proceder à leitura dos mesmos, a qual foi feita pela pesquisadora principal (ACAO) que aferia o maior diâmetro transverso da pápula formada com régua milimetrada, e sempre confirmada por outro participante do estudo (ES). Foram considerados positivos os testes cuja pápula de induração em seu diâmetro transverso apresentavase com tamanho acima de três milímetros em relação à pápula do controle negativo (solução glicerinada fisiológica a $0,9 \%) .21$

Para o banco de dados utilizou-se o programa Epi-info 6.0.

\section{Resultados}

Os indicadores sociais da comunidade evidenciaram nível de pobreza considerável, sendo a maioria dos informantes de baixa escolaridade, com ensino fundamental incompleto e condições de habitação, em geral, miseráveis (Tabela 1).

Para caracterizar clinicamente a asma entre as crianças de 6 a 10 anos foi considerada a terceira questão do formulário do ISAAC, 20 que indaga qual o número de crises apresentadas durante o último ano. Os resultados encontrados foram os seguintes: 
Tabela 1

Distribuição de freqüências das características socioeconômicas das crianças asmáticas de 6 a 10 anos residentes na comunidade do Campo do Banco-Várzea. Recife, Pernambuco, março de 1997 a junho de 1998.

\begin{tabular}{|c|c|}
\hline \multirow[t]{2}{*}{ Variáveis } & Freqüência \\
\hline & $\%$ \\
\hline
\end{tabular}

Idade

6 - 8 anos

$9-10$ anos

Total

67

56

123

Sexo

Masculino

Feminino

Total

72

51

123

58,5

41,5

100,0

Escolaridade materna

Não estudou

Fundamental incompleto

Fundamental completo

6

Outros (*)

Água

Encanada dentro de casa

101

Encanada fora de casa e outras

Total

22

123

Água para consumo

Tratada

Não tratada

108

108
15

Total

123

87,8

12,2

100,0

Moradia

Tijolo

Outros

Total

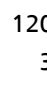

123

97,6

2,4

100,0

Destino dos dejetos

$\begin{array}{lll}\text { Sanitário com descarga } & 45 & 36,6\end{array}$

Sanitário sem descarga

Total

78

123

* No item escolaridade materna, em cinco questionários não foi possível obter esta informação.
Tabela 2

Distribuição de freqüências de positividade aos testes cutâneos para fungos em crianças com asma em atividade, residentes na comunidade de Campo do BancoVárzea. Recife, Pernambuco, março de 1997 a junho de 1998.

Extrato fúngico testado

\begin{tabular}{lrr}
\hline & N & $\%$ \\
\hline Aspergillus & 7 & 25,0 \\
Penicillium & 7 & 25,0 \\
Alternaria & 3 & 10,7 \\
Helminthosporium & 3 & 10,7 \\
Cladosporium & 3 & 10,7 \\
Mold mix & 5 & 17,9 \\
Total de testes & 28 & 100,0 \\
\hline
\end{tabular}

sem crise $(n=95,75,6 \%)$; uma a três crises $(n=15$, $13,0 \%)$, e quatro a 12 crises no último ano $(n=13$, $11,4 \%)$, entre as 123 crianças pesquisadas. Esse último grupo no qual foi verificada elevada freqüência de asma em atividade, ou seja, mais de três crises no último ano, constituiu a sub-amostra de 13 pacientes para o estudo de hipersensibilidade a fungos.

A distribuição de freqüências de positividade aos testes cutâneos aos respectivos extratos utilizados nas 12 crianças, encontra-se descrita na Tabela 2.

A hipersensibilidade a fungos no grupo de asmáticos em atividade ocorreu em 12 dos 13 escolares, cujos testes de hipersensibilidade imediata foram positivos a pelo menos um dos extratos fúngicos utilizados. Os resultados considerados positivos estão descritos em números que se referem ao diâmetro das pápulas, enquanto os testes negativos para os demais espécimes foram identificados com o símbolo "Ø", conforme pode ser visualizado na Tabela 3. Dentre os 12 pacientes "positivos", observou-se que dois apresentaram hipersensibilidade a apenas um dos extratos testados, sendo ambos sensíveis ao gênero Alternaria. Dentre os extratos fúngicos testados, o de maior positividade (em sete pacientes), foi o Aspergillus mix, seguido do Penicillium mix (em seis pacientes). 
Diâmetro de induração do teste cutâneo de hipersensibilidade imediata a extratos fúngicos segundo a ordem de inclusão dos 13 pacientes selecionados. Comunidade do Campo do Banco-Várzea. Recife, Pernambuco, março de 1997 a junho de 1998.

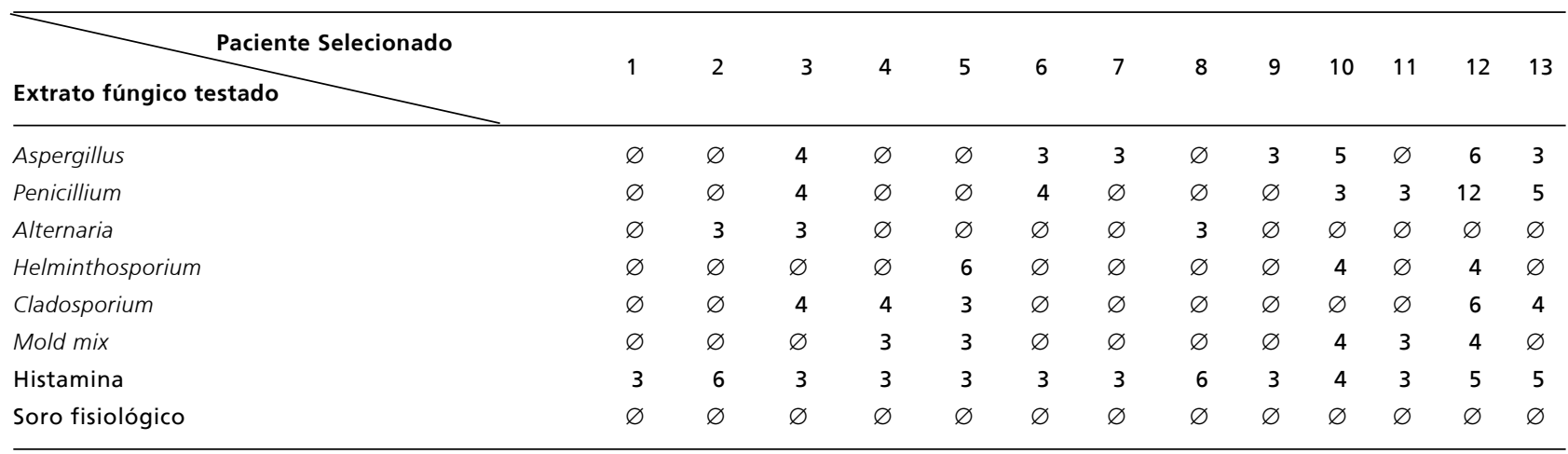

Induração: valores numéricos = resultado positivo; símbolo " $\varnothing$ " = resultado negativo

\section{Discussão}

O Nordeste brasileiro, especialmente nas regiões litorâneas, apresenta uma temperatura média elevada e condições de umidade que parecem constituir ambiente físico propício ao crescimento exacerbado de fungos, os quais podem atuar como importantes aeroalérgenos.22 Quando se estuda a hipersensibilidade a fungos, faz-se necessária a comprovação por testes in vivo, uma vez que a referência à presença ou não de mofo na residência constitui um dado inespecífico e que não deve ser valorizado isoladamente. 19

Fato interessante observado nesta pesquisa, é que a positividade aos testes cutâneos, em 12 das 13 crianças asmáticas, a pelo menos um dos extratos utilizados, condiz com os espécimes de fungos encontrados no ar ambiente domiciliar inicialmente obtidos pelo estudo piloto. 18 Entre os gêneros mais prevelentes estão os espécimes Aspergillus e Penicillium, em primeiro e terceiro lugares por ordem de freqüência. 18

Os extratos de fungos para testes cutâneos são complexos, havendo necessidade de padronização adequada. Conseqüentemente, acarreta limitações no estabelecimento de um ponto adequado de corte entre os vários extratos existentes, mas o mais aceito nos vários estudos é o valor de $3 \mathrm{~mm} \cdot{ }^{19-21}$

Uma limitação metodológica do presente estudo é que a positividade ao teste cutâneo de hipersensibilidade imediata associada às dosagens de $\operatorname{IgE}$ sérica total e específica para cada gênero, os quais não foi realizada.

Além disto, poderá constituir motivo de crítica a esta pesquisa o fato de não terem sido utilizados extratos de outros aeroalérgenos, como os ácaros. Em pesquisa anterior realizada em Recife, foi demonstrado que Blomia tropicallis e Dermatophagoides pteronyssinus são os ácaros mais sensibilizantes, com positividade acima de $90 \%$ em escolares asmáticos. Esta freqüência elevada pode ser atribuída à hipersensibilidade a fungos, que demonstram ser fator predisponente. 22

A exclusão dos pacientes com menor número de crises de dispnéia nos últimos 12 meses (menor ou igual a três), poderá constituir outro questionamento a essa pesquisa. No entanto, o referido grupo foi excluído para evitar erros de classificação da asma, quanto ao fato de o indivíduo ser ou não portador dessa condição, uma vez que em nosso meio é alta a prevalência de infecções respiratórias na infância, com risco de desenvolver dispnéia, 23 e não necessariamente asma. Contudo, os pacientes que apresentam mais de três crises nos últimos 12 meses atendem aos critérios clínicos para o diagnóstico de asma, a qual estando em atividade (com elevado número de crises) sugere maior probabilidade de tratar-se de asma extrínseca, relacionada a aeroalérgenos, como ácaros e fungos. ${ }^{24}$ Além disso, a sensibilização a fungos no paciente asmático é mais comum naqueles que desenvolvem quadros mais evidentes da doença, ${ }^{21}$ como pôde ser observado nos resultados.

Em estudo multicêntrico ${ }^{25}$ sobre a asma nos chamados "bolsões de pobreza", ou seja, nas comunidades de baixa renda das periferias de grandes cidades americanas, onde foram mensuradas as concentrações de fungos dentro e fora dos domicílios, foi demonstrado que quanto maior a umidade aliada à presença de gatos e baratas, maior 
a concentração de fungos no ar ambiente. Conseqüentemente, esses agentes constituem fator de risco para asma, inclusive com episódios fatais, 25 além de aumentarem o risco de sensibilização para outros alérgenos, tais como os ácaros. 26 Esses achados condizem com as características encontradas na maioria das residências das crianças, evidenciando a pobreza como fator agravante.

Apesar da existência dos fungos na Terra há cerca de 550 milhões de anos, tem sido levantada a suspeita de uma "nova doença" que atinge as pessoas tanto em domicílios quanto em prédios públicos, 27 decorrente da hipersensibilidade a esses agentes, a

\section{Referências}

1. Barnes K, Brenner R. Quality of housing and allergy to cockroaches in the Dominican Republic. Int Arch Allergy Immunol. 1996; 109: 68-72.

2. Wijnands LM, Deisz WD, van Leusden FM. Marker antigens to assess exposure to molds and their allergens. Allergy. 2000; 55: 850-5.

3. Helbling A. Animals and fungi as allergy inducers. Ther Umsch. 2001; 5: 97-303.

4. Bernard-Bonnin AC, Pelletier H, Allard-Dansereau C, Chabot G, Robert M, Masson P, Maheux B, Robitaille N. Parenteral knowledge about their asthmatic children. Pediatrie. 1991; 46: 489-97.

5. Dhillon M. Current status of mold immunotherapy. Ann Allerg. 1991; 66: 385-92

6. Tabar AI, Lizaso MT, Garcia BE, Echechipia S, Olaguibel JM, Rodriguez A. Tolerance of immunotherapy with an alternaria extract in patients with rhinitis and bronchial asthma. J Investig Allergol Clin Immunol. 2000; 10: 32733.

7. Bonifazi F. Immunotherapy in pollen and mold asthma. Monaldi Arch Chest Dis. 1994; 49: 150-3.

8. Yang X. Does allergen immunotherapy alter the natural course of allergic disorders? Drugs. 2001; 61: 365-74.

9. Guneser S, Atici A, Koksal F, Yaman A. Mold allergy in Adana, Turkey. Allergol Immunopathol. 1994; 22: 52-4.

10. Horak B, Dutkiewicz J, Solarz K. Microflora and acarofauna of bed dust from homes in Upper Silesia, Poland. Ann Allergy Asthma Immunol. 1996; 76: 41-50.

11. Escudero AI, Sanchez-Guerrero IM, Mora AM, Soriano V, Lopez JD, Garcia FJ, Negro JM, Hernandez J, Pagan JA. Cost effectiveness of various methods of diagnosing hypersensitivity to Alternaria. Allergol Immunopathol. 1993; 21 : 153-7.

12. Malling HJ, Dreborg S, Weeke B. Diagnosis and immunotherapy of mold allergy. Allergy. 1987; 42: 305-14.

13. Yamaguchi H. Evaluation of immediate hypersensitivity and environmental factors by intracutaneous skin test and test specific IgE antibodies in allergic children. Arerugi. 1993; 42: 571-81. qual deverá ser estabelecida pela história clínica, pelo exame físico e por testes adequados nos casos suspeitos. 27 Nesta busca será importante a determinação específica de "qual" fungo é relevante, de "como" ocorre a exposição e que quantidade de esporos é suficiente para desencadear o processo. 27

Embora a presente pesquisa tenha sido realizada para explorar a possibilidade de que a hipersensibilidade a fungos seja relevante no nosso meio, serão necessários estudos analíticos adicionais sobre o tema, pois se trata de assunto importante e atual no estudo da asma.

14. Faria A. Aspectos ecológicos e clínicos da flora micótica anemófila de Belo Horizonte [tese doutorado]. Belo Horizonte: Faculdade de Medicina da Universidade Federal de Minas Gerais; 1967.

15. Lacaz CS, Porto E, Martins JEC. Fungos, actimomicetos e algas de interesse médico. In: Lacaz CS. Micologia médica. 8. ed. São Paulo: Sarvier; 1991. Cap. 32, p. 483-90.

16. Alecrim I, Teixeira H. Fungos anemófilos na cidade do Recife (Pernambuco, Brasil). An. Fac Med Univ Recife. 1958; 18: 269-74.

17. Machado GMR. Fungos anemófilos de áreas do Grande Recife [dissertação mestrado]. Recife: Centro de Ciências da Saúde do Departamento de Medicina Tropical, Universidade Federal de Pernambuco; 1979.

18. Osório ACA, Sarinho ESC, Baptista EVP, Lacerda Filho NA. Incidência de fungos no ar em residências de crianças asmáticas. In: Anais do V Congresso Nacional de Iniciação Científica; 1997 out. 8 a 10; Recife, Pernambuco. Recife: Ed. Universidade Federal de Pernambuco; 1997. p. 73.

19. Li JT, Andrist D, Bamlet WR, Wolter TD. Accuracy of patient prediction of allergy skin test results. Ann Allergy Asthma Immunol. 2000; 85: 382-4.

20. Asher MI, Keil U, Anderson HR, Beasley R, Crane J, Martinez F, Mitchell EA, Pearce N, Sibbald B, Stewart AW, Strachan D, Weiland SK, Williams HC. International Study of Asthma and Allergies in Childhood (ISAAC): rationale and methods. Eur Respir J. 1995; 8: 483-91.

21. Rosário Filho NA, Brom AL, Perrini JC, Arruda LK, Bernd LAG, Geller M, Mendes NF, Aun WT. Comissão de testes, imunoterapia e padronização de antígenos: testes cutâneos em alergia. Rev.Bras Alerg Imunopatol. 2000; 23: 134-6.

22. Sarinho E. Hipersensibilidade aos ácaros da poeira domiciliar em pacientes asmáticos: estudo de caso-controle em escolares [tese doutorado]. Recife: Departamento MaternoInfantil do Centro de Ciências da Saúde da Universidade Federal de Pernambuco; 1998.

23. Huss K, Adkinson NF Jr, Eggleston PA, Dawson C, Van Natta ML, Hamilton RG. House dust mite and cockroach exposure are strong risk factors for positive allergy skin test responses in the childhood. J Allergy Clin Immunol. 2001; 107: 48-54. 
24. Ross MA, Curtis L, Scheff PA, Hryhorczuk DO, Ramakrishnan V, Wadden RA, Persky VW. Association of asthma symptoms and severity with indoor bioaerosols. Allergy. 2000; 55: 705-11.

25. O'Connor GT, Walter M, Mitchell H, Kattan M, Morgan WJ, Gruchalla RS, Pongracic JA, Smartt E, Stout JW, Evans $\mathrm{R}$, Crain EF, Burge HA. Airborne fungi in the homes of children with asthma in low-income urban communities: the Inner-City Asthma Study. J Allergy Clin Immunol. 2004; 111: 599-606.

Recebido em 30 de outubro de 2002

Versão final apresentada 12 de maio de 2006

Aprovado em 25 de maio de 2006
26. Lemanske RF. Issues in understanding pediatric asthma: epidemiology and genetics. J Allergy Clin Immunol. 2002; 109 (Suppl 6): S521-4.

27. Terr AI. Are indoor molds causing a new disease? J Allergy Clin Immunol. 2004; 113: 221-6. 